International Journal of Engineering \& Technology, $7(4.4)(2018) 38-39$
International Journal of Engineering \& Technology
SPC
Website: www.sciencepubco.com/index.php/IJET
Research paper

\title{
UX Design for the Visually Impaired to Improve Health Information Accessibility
}

\author{
Woo Jin Kim, Min Ji Kim and Il Kon Kim* \\ ${ }^{1}$ Computer Science and Engineering ,Kyoungpook National University (South Korea)
}

\begin{abstract}
This study was conducted on individuals with low vision, who constitute the highest population among visual impairment patients. We conducted an experiment to determine whether there was an improvement in accessibility to visual information for the visually impaired after applying the UX design guidelines. Additionally, we analyzed whether this UX design affects the information recognition rate for the non-disabled individuals.
\end{abstract}

Keywords:Mobile App Accessibility, Impaired Informatics.

\section{Introduction}

Mobile Web 2.0, which developed along with the evolution of Web 2.0, has brought opportunities for the visually impaired to participate in social activities, including communication with nondisabled people, and economic activities.

However, the development of health apps involves following several design guidelines provided by Apple and Google, but app planners, developers, and designers are generally not provided with specific guidelines for the visually impaired or for those with other special needs.

Therefore, to solve this problem, 1) we identified the cognitive behavior of individuals with low vision and 2) we conducted an experiment to check whether the UX design guidelines actually improve the accessibility to visual information, by using a selfdeveloped UX design evaluation app.

\section{Method}

\subsection{Cognitive Behavior of Individuals with Low Vision}

In this study, myopia, tunnel vision, and color vision abnormality were defined as representative low vision, and subjects who face difficulties in accessing visual information on mobile devices were selected for the study.

Table 1:. Major Aspects of Visual Information Cognition in the Impaired Major Visual Information Cognition of the Visually Impaired

1) When the visual information is coming, amblyopia patients try to keep the physical distance very close between the subject and the eye.

2) Due to the scope of vision, recognition of long letters or images on one side is impaired.

3 ) If the direction of the eye movement changes because of diplopia, it takes time to recognize the information momentarily.

4) When the visually impaired read something, they read along the line of sight with the fingers and other reference points.

5) It is difficult to find specific information from information distributed at

irregular intervals.
The smaller the shade is, the more difficult it is to recognize information.
6) People who have color vision abnormality cannot distinguish between
colors with the same level of saturation.
7) Acquiring information through letters seems more tiring than through
images.
8) There is the difference in the acquisition of information according to the
brightness of the light source.
9) If the mobile screen which they are viewing changes often, it is difficult
to acquire the information and they predict the next screen by the sound.
10) The subject's focus often changes or appears dimmed. Astigmatism
leads to distorted or blurred vision.

\subsection{UX Design Guidelines for the Visually Impaired}

Here, we focused on visual communication design to improve visual information accessibility of mobile health apps for individuals with low vision, and conducted a UX design study.

We analyzed UX design guidelines that were developed based on the cognitive patterns of the visually impaired, as discussed above. The guidelines were developed by taking W3C's Web Content Accessibility Guidelines (WCAG) as the basic framework.

\subsection{Mobile Visual Information Accessibility Verifica- tion Experiment Design for Individuals with Low Vision}

This study conducted an information accessibility test of the health apps for 17 individuals with low vision (Group A). The test was conducted by comparing 1) the current status of health apps and 2) the status after applying the UX design guidelines.
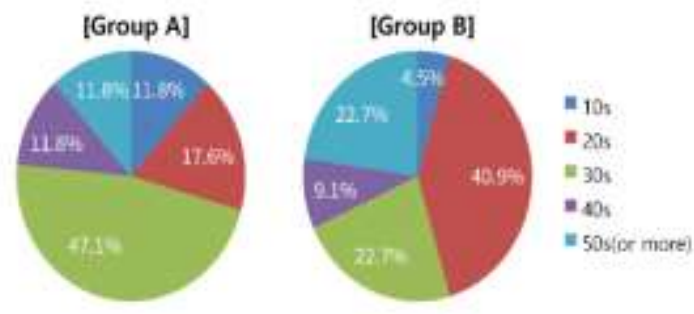

Figure 1:. Age range of participants in each group 


\section{Results}

This study consists of 10 pages, with each page containing a question (in total, 10 pages and 10 questions). In addition, the study was divided into two parts: Part $1(\mathrm{Q} 1 \sim \mathrm{Q} 5)$ comprised screens of theexisting health apps and Part 2 (Q6 Q10) comprised screens that were designed based on the UX design guidelines.

For Group A (individuals with low vision), Part 2, in which the UX design guidelines were applied, an improvement in visual information recognition speed of $13.68 \%$ was observed, compared with Part 1; moreover, the information was recognized approximately $10.78 \mathrm{~s}$ faster than the average speed.

For Group B (individuals with no disabilities), contrary to the expectation before the experiment, visual information recognition speed in Part 2 was higher than in Part 1, which was the same for Group A.

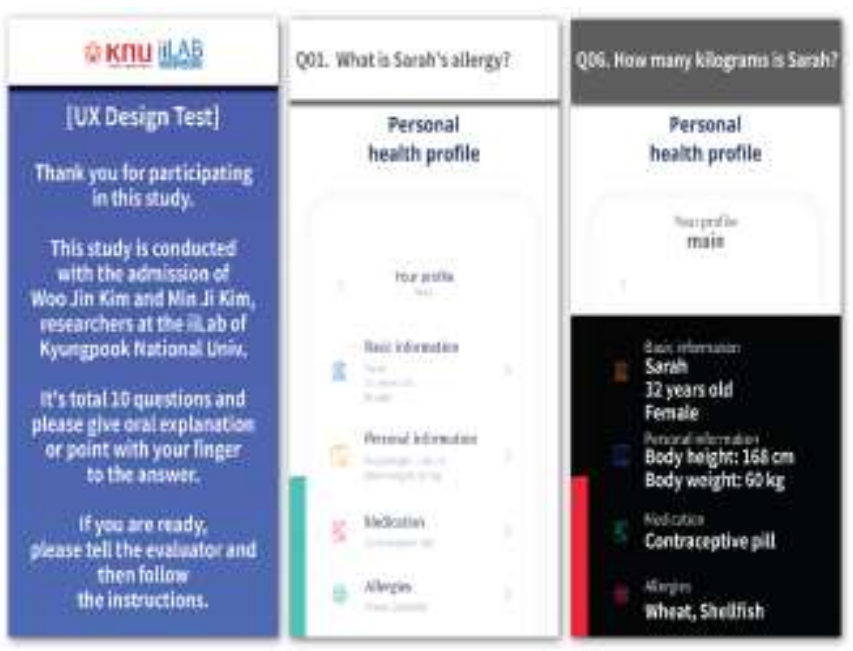

Figure 2:. UX test app screens used in the experiments

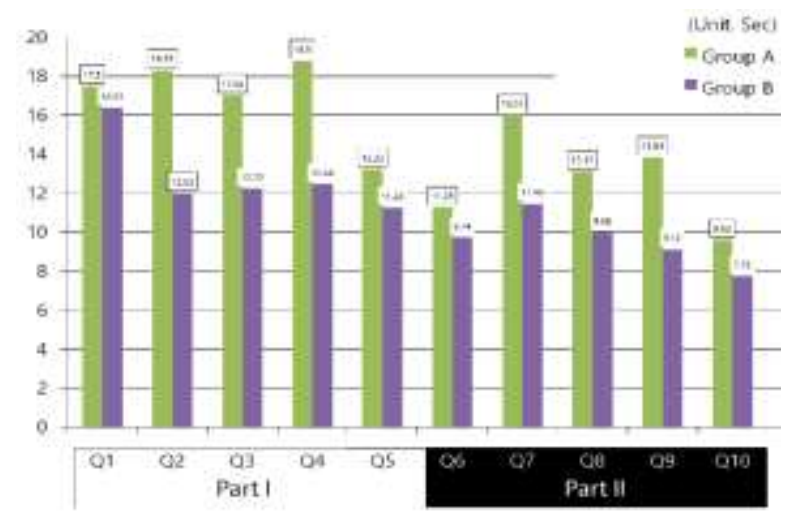

Figure 3:. Group A\& B response time and correct answer rate

When analyzing the overall results of this experiment, it was clear that there are differences of information recognition between Group A and Group B. That is, when the same information is provided, the gap between groups is more than $32.41 \%$.

\section{Discussion \& Conclusion}

The greatest limitation of this experiment was the difficulty in finding subjects who had been identified as individuals with low vision. For example, among the individuals with low vision who participated in this experiment, there were three RP patients, and the incidence of this disease is only 1 in 5,000.
In addition, for experiments with a small sample, as in this study, it is necessary to admit the local limitations and search for ways to conduct multinational collaborative research.

Health apps are a significant help for obtaining users' healthcare information from healthcare providers by managing and sharing the recorded information, and not just only for recording personal health information.

Notably, this study focuses solely on the methodology of delivering visual information. Therefore, there is a possibility that consideration of aesthetic design elements required by modern society is insufficient.

Owing to these reasons, UX design study on intuitive visual information transmission method satisfying users' aesthetic sense should be followed.

\section{Acknowledgment}

Research supported by the Technology Innovation Program (10053249, Development of Personalized Healthcare System exploiting User Life-Log and Open Government Data for Business Service Model Proof on Whole Life Cycle Care) ) funded By the Ministry of Trade, industry \& Energy(MI, Korea)

\section{References}

[1] Ai SATO, Mia MATSUO, Elsuke YOSHIMURA, Mle NAKAHARA, An Increasingly Aging World and Expectations for the Medical Device Industry, NRI Papers(2015).

[2] Chuck Martin, "The Third Screen: Marketing to Your Customers in a World Gone Mobile", Massachusetts, 2011.

[3] Ericsson, "Ericsson Mobility Report", Ericsson, Stockholm, Sweden, 2017

[4] Federal Communications Commission, "Twenty-First Century Communications and Video Accessibility Act", Columbia, 2010.

[5] Peter LaBerge, "The 2017 Mobile Growth Handbook", branch, California, 2017.

[6] Research2Guidance, "mHealth Economics 2017 - Current Status and Future Trends in Mobile Health" Research2Guidance, Berlin, Germany, 2017. 